\title{
Estudo retrospectivo de 207 casos de tumores mamários em gatas ${ }^{1}$
}

\author{
Monique Togni $^{2 *}$, Eduardo K. Masuda ${ }^{3}$, Glaucia D. Kommers ${ }^{4}$, Rafael A. Fighera ${ }^{4}$ \\ e Luiz Francisco Irigoyen ${ }^{4}$
}

\begin{abstract}
Togni M., Masuda E.K., Kommers G.D., Fighera R.A. \& Irigoyen L.F. 2013. [A retrospective study of $\mathbf{2 0 7}$ cases of mammary tumors in queens.] Estudo retrospectivo de 207 casos de tumores mamários em gatas. Pesquisa Veterinária Brasileira 33(3):353358. Departamento de Patologia, Universidade Federal de Santa Maria, Camobi, Santa Maria, RS 97105-900, Brazil. E-mail: moniquetogni@yahoo.com.br

This study aimed to determine the most prevalent mammary tumors in cats and associate them to some prognostic factors. The files from the Laboratório de Patologia Veterinária (LPV) of the Universidade Federal de Santa Maria (UFSM) were reviewed, and 1.427 feline biopsies and autopsy protocols between the years 2000 and 2011 were found. Based on the information retrieved from the files, a relationship was established among the tumors and some prognostic factors such as sex, age, breed, reproductive status, use of contraceptives, number and location of affected glands, ulcers, size of the neoplasm, distant metastases, and affected lymph nodes. Thus, it was observed that mammary cancer is the second most prevalent diagnosis, following skin tumors. All cats with mammary tumors were female, being the elderly and mixed breed the most affected. Malignant neoplasms were the most frequently diagnosed, followed by non-neoplastic tumors, and benign neoplasms. Smaller tumors were mostly carcinomas. Ulcerations were present not only in malignant neoplasms but also in non-neoplastic changes. Distant metastases were found mainly to the lungs and skin.

INDEX TERMS: Mammary gland, mammary neoplasms, tumors in cats.
\end{abstract}

RESUMO.- Este estudo teve como objetivos determinar os tumores mais prevalentes em gatos e relacionar os tumores mamários a alguns de seus fatores prognósticos. Os arquivos do Laboratório de Patologia Veterinária (LPV) da Universidade Federal de Santa Maria (UFSM) foram revisados e um total de 1.427 protocolos de biopsias e necropsias de felinos, entre 2000 e 2011, foi encontrado. Com base nas informações dos arquivos, foi estabelecida a relação entre os tumores e alguns fatores como sexo, idade, raça, estado reprodutivo, uso de contraceptivos, número e localização das glândulas afetadas, ulcerações, tamanho do neoplasma, metástases distantes e para os linfonodos. Assim, ob-

\footnotetext{
${ }^{1}$ Recebido em 2 de outubro de 2012.

Aceito para publicação em 3 de dezembro de 2012.

Parte da Dissertação de mestrado do primeiro autor.

${ }^{2}$ Programa de Pós-Graduação em Medicina Veterinária, área de concentração em Patologia Veterinária, Centro de Ciências Rurais, Universidade Federal de Santa Maria (UFSM). Av. Roraima 1000, Santa Maria, RS $97105-$ 900, Brasil. *Autor para correspondência: moniquetogni@yahoo.com.br

${ }^{3}$ Médico Veterinário autônomo, Avenida do Forte 557, sala 203, Porto Alegre, RS 91360-000, Brasil.

${ }^{4}$ Departamento de Patologia, UFSM, Av. Roraima 1000, Santa Maria, RS 97105-900.
}

servou-se que os tumores de mama foram o segundo diagnóstico mais prevalente, após os tumores de pele. Todos os gatos com tumores mamários eram fêmeas, sendo os sem raça definida e os idosos os mais afetados. Os neoplasmas malignos foram diagnosticados com maior frequência, seguidos pelos tumores não neoplásicos e pelos neoplasmas benignos. Os tumores menores eram, na sua maioria, carcinomas. Ulcerações estavam presentes não só em neoplasmas malignos, mas também em alterações não neoplásicas. Metástases distantes foram encontradas principalmente nos pulmões e na pele.

TERMOS DE INDEXAÇÃO: Glândula mamária, neoplasias mamárias, tumores em felinos.

\section{INTRODUÇÃO}

O número de atendimentos, exames e necropsias de gatos tem crescido consideravelmente nos últimos anos no LPV-UFSM, sendo esse aumento de 61 para 172 casos entre os anos de 2000 e 2011, respectivamente.

Tumores mamários são comuns em cães e gatos, mas raros em outras espécies animais, sendo que somente para essas duas espécies, há uma classificação especial (Hampe 
\& Misdorp 1974). Há numerosos estudos sobre tumores de mama em cadelas, porém ainda há poucos estudos relacionados a tumores de mama em gatas. Por apresentarem etiologia, patogenia, fatores prognósticos (Hayes et al. 1981, Hellmén et al. 1993, Weijer \& Hart 1983) e histologia (Misdorp \& Weijer 1980, Pérez-Alenza et al. 2004) semelhantes aos da mulher, estudos sobre tumores mamários em gatas são de grande interesse na pesquisa do câncer de mama em humanos (Hayes et al. 1981, Weijer \& Hart 1983, Hellmén et al. 1993).

Tamanho tumoral, tipo de tratamento cirúrgico, envolvimento de linfonodos e tipo de crescimento são fatores clinicopatológicos bem estabelecidos. Entretanto, associações entre aspectos clínicos, epidemiológicos e histopatológicos foram pouco descritas (Misdorp 2002). Dessa forma, o objetivo do presente trabalho foi organizar os dados recolhidos dos arquivos do LPV-UFSM, a fim de estabelecer os tumores mais prevalentes em gatos e as relações entre os tumores mamários e alguns de seus fatores prognósticos, auxiliando assim clínicos e patologistas na escolha terapêutica adequada e no estabelecimento de um prognóstico mais fidedigno.

\section{MATERIAL E MÉTODOS}

Os protocolos compreendidos entre os anos de 2000 e 2011 do arquivo do LPV-UFSM foram revisados, sendo encontrados 1.427 protocolos de felinos. Todos os tumores mamários classificados como neoplásicos (malignos ou benignos) e não neoplásicos (hiperplasias ou displasias) foram incluídos neste estudo.

Do histórico clínico foram avaliados, quando constavam, a idade, sexo, raça, estado reprodutivo e o uso de contraceptivos. Os animais foram classificados quanto à faixa etária em jovens (menos de ano), adultos (de um a oito anos) e idosos (acima de oito anos de idade).

Das variáveis clinicopatológicas, foram estudadas as mamas afetadas, a presença de ulcerações cutâneas, metástases (distantes e em linfonodos) e o tamanho do tumor. As cadeias mamárias foram classificadas em dois grupos, de acordo com o padrão de drenagem linfática existente nessas regiões. 0 primeiro grupo (G1) compreende as mamas torácicas e as abdominais craniais. O segundo (G2), é composto pelas mamas abdominais caudais e inguinais. Com relação ao tamanho, esses foram classificados de acordo com o sistema TNM (tamanho, envolvimento de linfonodos e metástases distantes), proposto pela Organização Mundial de Saúde (OMS), pelo qual tumores menores que $3 \mathrm{~cm}$ são classificados como T1, entre 3 e $5 \mathrm{~cm}$ como T2 e acima de $5 \mathrm{~cm}$ classificados como T3. Quando havia mais de um tumor, cada um foi mensurado e avaliado separadamente. Para a avaliação das metástases distantes, apenas foi considerada ausência ou presença de metástases, seja por avaliação clínica, biópsias ou necropsias. 0 mesmo foi aplicado na avaliação dos linfonodos regionais mamários e para as ulcerações cutâneas.

Quanto à avaliação histopatológica, consideraram-se apenas os diagnósticos morfológicos presentes nos protocolos, subclassificando-os pelo sistema de classificação vigente do Instituto de Patologia das Forças Armadas - Organização Mundial da Saúde (Misdorp et al. 1999) quando julgou-se necessário. Foram avaliados todos os diagnósticos morfológicos dos protocolos, mesmo quando estes diagnósticos estavam presentes mais de uma vez. Quando isso ocorreu, cada diagnóstico foi considerado separadamente obtendo-se, ao final, número maior de tumores do que de gatas.

\section{RESULTADOS}

Após a revisão de todos os protocolos de biópsias e necropsias de gatos, durante o período analisado, foram encontrados 1.427 casos, $53 \%$ (753/1427) de biópsias e $47 \%$ (674/1427) de necropsias. Desses, 36,65\% (523/1.427) são protocolos com tumores, sendo os cutâneos, com $41,49 \%(217 / 523)$, os mais prevalentes, seguidos dos tumores mamários com 39,57\% (207/523) e dos hemolinfopoéticos, com 6,50\% (34/523). Os 12,42\% (65/523) restantes são tumores dos outros sistemas. A prevalência dos tumores mamários de gatas com em relação ao total de protocolos desta espécie, durante o período analisado, foi de $14,50 \%$ (207/1.427), sendo 92,75\% (192/207) oriundas de biópsias e 7,24\% (15/207) de necropsias.

Foram encontrados 217 tumores mamários, em 207 gatas, incluindo tumores neoplásicos e não neoplásicos. 0 maior número de tumores com relação ao número de gatas deve-se ao fato de que 10 gatas apresentaram mais de um tipo de tumor mamário.

Todos os animais eram fêmeas, sendo que 9,67\% (20/207) dos protocolos mencionaram a castração das gatas e $23,19 \%$ (48/207) afirmaram que as fêmeas eram inteiras. Nos demais casos, 67,14\% (139/207), não havia informações suficientes para se determinar o estado reprodutivo das gatas. Constatou-se que $26,08 \%$ das gatas afetadas receberam fármacos contraceptivos previamente (Quadro 1). Porém, não havia informações referentes à idade da primeira administração, frequência, tipo e dose do fármaco utilizado.

Dentre as raças mais acometidas, cinco foram identificadas. Os animais sem raça definida foram os mais comumente diagnosticados com 54,10\% (112/207), seguidos pelas raças Siamesa com 25,60\% (53/207), Persa com $7,73 \%$ (16/207), Himalaia com 2,42\% (5/207) e Angorá com 0,97\% (2/207). Em 9,18\% (19/207) dos protocolos a raça não foi informada conforme o Quadro 1.

Quadro 1. Prevalência epidemiológica das gatas acometidas por tumores mamários no LPV-UFSM (2000-2011)

\begin{tabular}{|c|c|c|}
\hline & $\begin{array}{c}\text { № de gatas } \\
\text { afetadas }\end{array}$ & $\begin{array}{c}\text { Frequência } \\
(\%)\end{array}$ \\
\hline \multicolumn{3}{|l|}{ 1. $\operatorname{Sexo}^{\mathrm{a}}$} \\
\hline 1.1 Macho & - & - \\
\hline 1.2 Fêmeas & 207 & 100 \\
\hline \multicolumn{3}{|l|}{ 2. $\operatorname{Raça}^{\mathrm{a}}$} \\
\hline 2.1 SRD & 112 & 54,10 \\
\hline 2.2 Siamês & 53 & 25,60 \\
\hline 2.3 Persa & 16 & 7,73 \\
\hline 2.4 Himalaia & 5 & 2,42 \\
\hline 2.5 Angorá & 2 & 0,97 \\
\hline 2.6 N.I. $^{\mathrm{b}}$ & 19 & 9,18 \\
\hline \multicolumn{3}{|l|}{ 3. Idade $\mathrm{I}^{\mathrm{a}}$} \\
\hline 3.1 Idosos & 119 & 57,49 \\
\hline 3.2 Adultos & 60 & 28,99 \\
\hline 3.3 Jovens & 9 & 4,34 \\
\hline 3.4 N.I. $^{\mathrm{b}}$ & 19 & 9,18 \\
\hline \multicolumn{3}{|l|}{ 4. Estágio reprodutivo ${ }^{\mathrm{a}}$} \\
\hline 4.1 Inteiras & 48 & 23,19 \\
\hline 4.2 Castradas & 20 & 9,67 \\
\hline 4.3 N.I. $^{\mathrm{b}}$ & 139 & 67,14 \\
\hline 5. Uso de contraceptivo ${ }^{a}$ & 54 & 26,08 \\
\hline
\end{tabular}


A idade das gatas com tumores mamários variou de 6 meses a 20 anos (média de 8 anos e 6 meses \pm 3 anos e 9 meses), observando-se maior frequência nas idosas, seguido das adultas e das jovens (Quadro 1). Dentre as gatas jovens, apenas $11,11 \%(1 / 9)$ apresentavam neoplasia, sendo o restante, acometido por alterações não neoplásicas. A idade de 9,18\% (19/207) dos felinos não foi informada.

Na análise das glândulas mamárias, as mais afetadas foram às torácicas e/ou abdominais craniais (G1) e o restante foi igualmente distribuído entre as gatas que apresentaram tumor nas mamas abdominal caudal e/ou inguinal (G2) e múltiplos nódulos em G1 e G2 (Quadro 2). Dos grupos G1

Quadro 2. Prevalência das variáveis clinicopatológicas de gatas acometidas por tumores mamários no LPV-UFSM (2000-2011)

\begin{tabular}{lcc}
\hline & $\begin{array}{c}\text { No de gatas } \\
\text { afetadas }\end{array}$ & $\begin{array}{c}\text { Frequência } \\
(\%)\end{array}$ \\
\hline 1. Grupo mamário & \\
1.1 G1 & 44 & 21,25 \\
1.2 G2 & 26 & 12,56 \\
1.3 G1 e G2 & 26 & 12,56 \\
1.4 N.I. & 111 & 53,63 \\
2. Ulcerações & 50 & 24,15 \\
2.1 Neoplasma maligno & 46 & 92 \\
2.2 Alteração não neoplásica & 4 & 8 \\
3. Metástases & & \\
3.1 Linfonodos & 15 & 7,24 \\
3.1.1 C. túbulo-papilar & 10 & 66,7 \\
3.1.2 C. cribriforme & 3 & 20 \\
3.1.3 Carcinossarcoma & 1 & 6,65 \\
3.3.4 Carcinoma sólido & 1 & 6,65 \\
3.2 Distantes & 13 & 6,28 \\
3.2.1 C. túbulo-papilar & 8 & 61,53 \\
3.2.2 C. cribriforme & 5 & 38,47 \\
4. Tamanho do tumor & & \\
4.1 T1 & 94 & 43,32 \\
4.2 T2 & 42 & 19,35 \\
4.3 T3 & 49 & 14,75 \\
4.4 N.I. & 32 &
\end{tabular}

${ }^{a}$ Dados avaliados em 207 gatas. ${ }^{b}$ N.I. $=$ não informado.${ }^{c}$ Dados avaliados em 217 tumores: G1 = glândula, mamária torácica e abdominal cranial, G2 = glândula mamária abdominal caudal e inguinal.

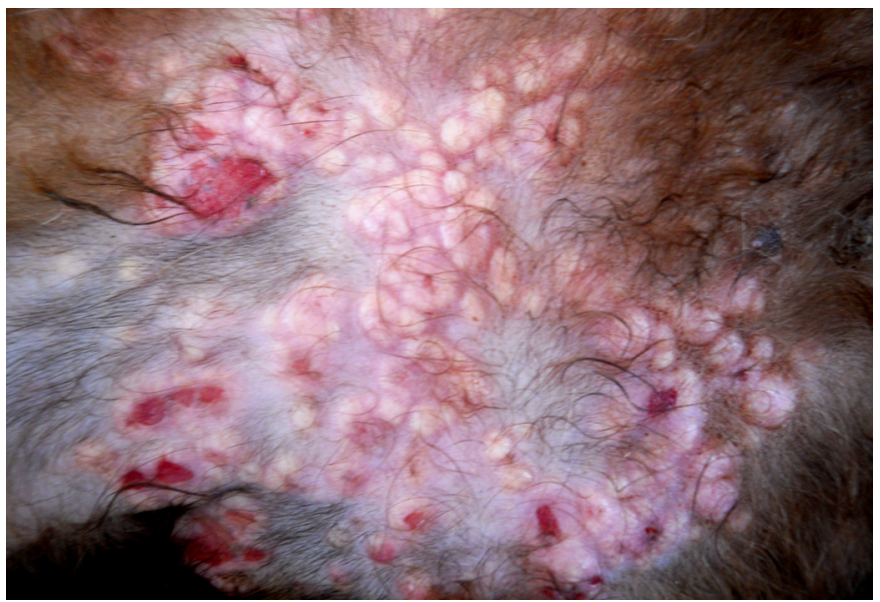

Fig.1. Metástase na pele de carcinoma túbulo-papilar. Há pequenos nódulos multifocais a coalescentes, alguns deles ulcerados.
Quadro 3. Prevalência dos diagnósticos morfológicos de tumores mamários de gatas no LPV-UFSM (2000-2011)

\begin{tabular}{lcc}
\hline & $\begin{array}{c}\text { № de gatas } \\
\text { afetadas }\end{array}$ & $\begin{array}{c}\text { Frequência } \\
(\%)\end{array}$ \\
\hline 1. Tumores malignos* & 157 & 72,35 \\
1.1 Carcinoma & 154 & 98,08 \\
1.1.1 C. túbulo-papilar & 86 & 55,84 \\
1.1.2 C. cribriforme & 47 & 30,52 \\
1.1.3 C. sólido & 20 & 12,99 \\
1.1.4 C. in situ & 1 & 0,65 \\
1.2 Carcinossarcoma & 3 & 1,91 \\
2. Tumores benignos* & 11 & 5,06 \\
2.1 Adenoma simples & 7 & 63,64 \\
2.2 Fibroadenoma & 2 & 18,18 \\
2.3 Adenoma complexo & 1 & 9,09 \\
2.4 Tumor misto benigno & 1 & 9,09 \\
3. Alterações não neoplásicas* & 49 & 22,58 \\
3.1 Hiperplasia Fibroepitelial & 29 & 59,18 \\
3.2 Displasia cística & 12 & 24,49 \\
3.3 Hiperplasia ductal & 5 & 10,21 \\
3.4 Ectasia ductal & 3 & 6,12 \\
* Dados avaliados em 217 tumores. & &
\end{tabular}

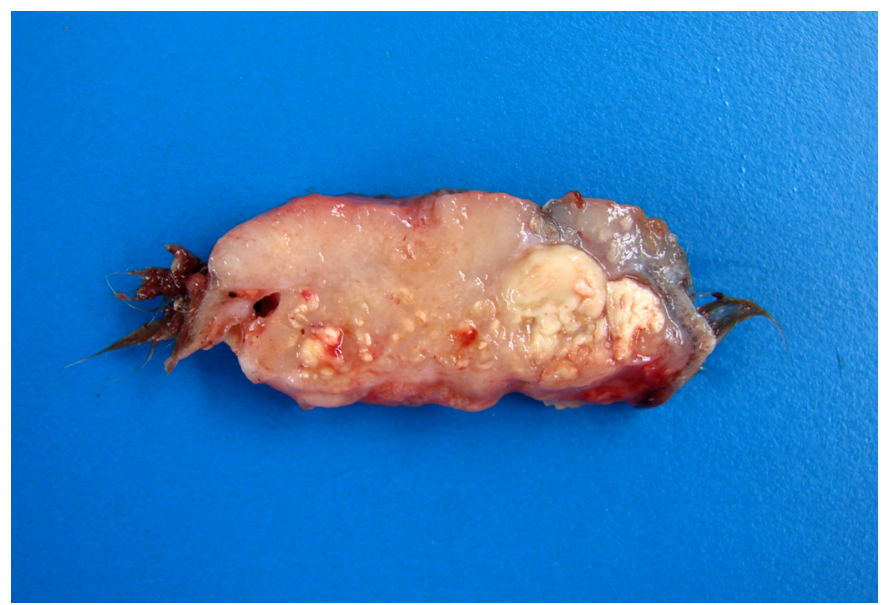

Fig.2. Superfície de corte de um carcinoma cribriforme mamário. São observadas áreas multifocais brancacentas e friáveis que correspondem às áreas de necrose comumente evidenciadas neste tipo de neoplasma.

e G2, em 21,73\% (45/207) dos casos apenas uma glândula foi afetada. Nos demais protocolos, 53,63\% (111/207), não havia informações suficientes para determinar a localização do tumor. Ulceração foi observada em 24,15\% (50/207), das gatas, sendo encontradas em neoplasias malignas e alterações não neoplásicas (Quadro 1). Dentre os neoplasmas malignos ulcerados, o carcinoma túbulo-papilar foi o mais prevalente com 54,34\% (25/46). Com relação às alterações não neoplásicas, todas as que apresentavam ulceração eram hiperplasias fibroepiteliais.

Na avaliação dos linfonodos, foram encontrados metástases de carcinoma túbulo-papilar, carcinoma cribriforme, carcinoma sólido e de carcinossarcoma. Nas metástases distantes foram observados apenas carcinoma túbulo-papilar e carcinoma cribriforme (Quadro 2). Dentre os locais de metástases distantes o pulmão com 76,92\% (10/13) foi o mais acometido, seguido da pele (Fig.1) 23,07\% (3/13), fígado e pleura ambos com 15,38\% (2/13). Além disso, o 
baço, peritônio, ossos e músculos esqueléticos também foram acometidos, porém com apenas 7,69\% (1/13) cada. Em seis casos mais de um órgão foi acometido.

Avaliando o tamanho, os tumores classificados como T1 foram os mais encontrados, seguidos de T3 e T2 (Quadro 2). Dos 43,31 \% dos tumores classificados como T1, $82,97 \%$ (78/94) eram carcinomas.

Quanto ao diagnóstico morfológico (Quadro 3), os neoplasmas malignos foram os mais prevalentes, seguidos das alterações não neoplásicas e dos neoplasmas benignos. A relação benigno:maligno para os neoplasmas foi de 1:14,27. Dos neoplasmas malignos, o carcinoma foi o mais prevalente seguido de carcinossarcoma. Dos carcinomas, o padrão túbulo-papilar foi o mais comum, seguido do padrão cribriforme (Fig.2 e 3), do sólido e do carcinoma in situ. Dos neoplasmas benignos o adenoma simples o mais prevalente, seguido de fibroadenoma, adenoma complexo e tumor misto benigno. Nas alterações não neoplásicas a hiperplasia fibroepitelial (Fig.4 e 5) foi a mais prevalente, seguida de displasia cística (Fig.6), hiperplasia ductal e de ectasia ductal.

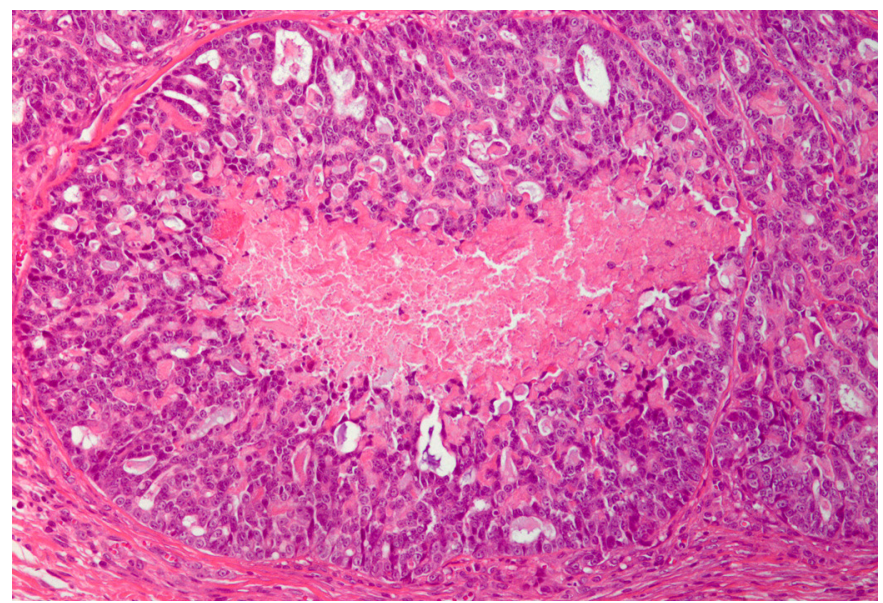

Fig.3. Aspecto histológico de um carcinoma cribriforme. Observa-se uma área de necrose central circundada por células epiteliais formando pequenas aberturas semelhantes a uma peneira. HE, obj.20x.

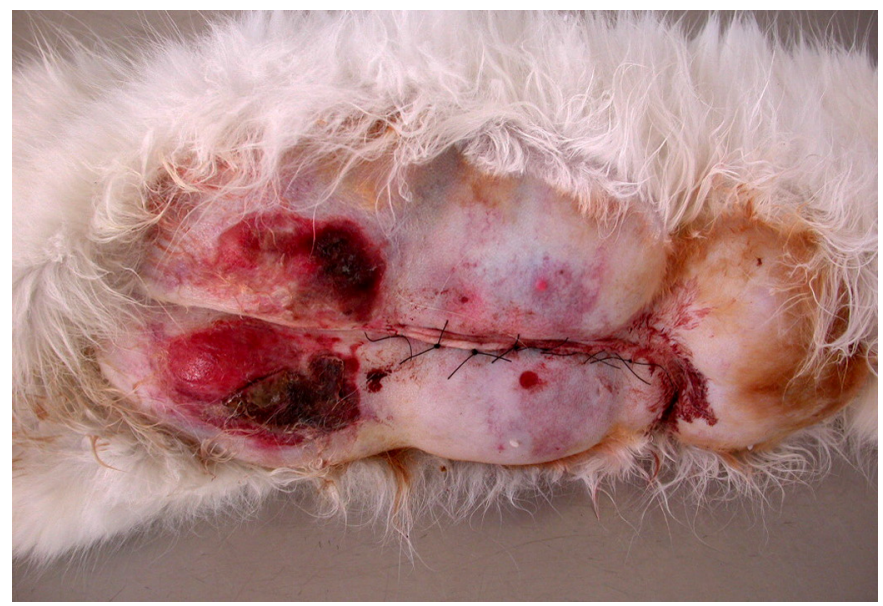

Fig.4. Gata com hiperplasia fibroepitelial. Observa-se aumento de volume acentuado de todas as mamas.

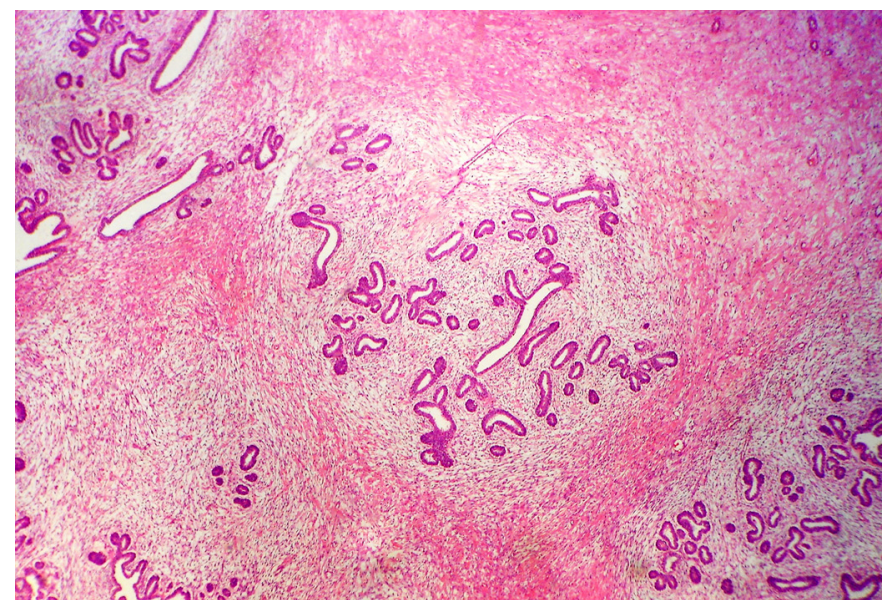

Fig.5. Aspecto histológico da hiperplasia fibroepitelial. Observa-se a proliferação ductal circundada por acentuado estroma fibroso. HE, obj.10x.

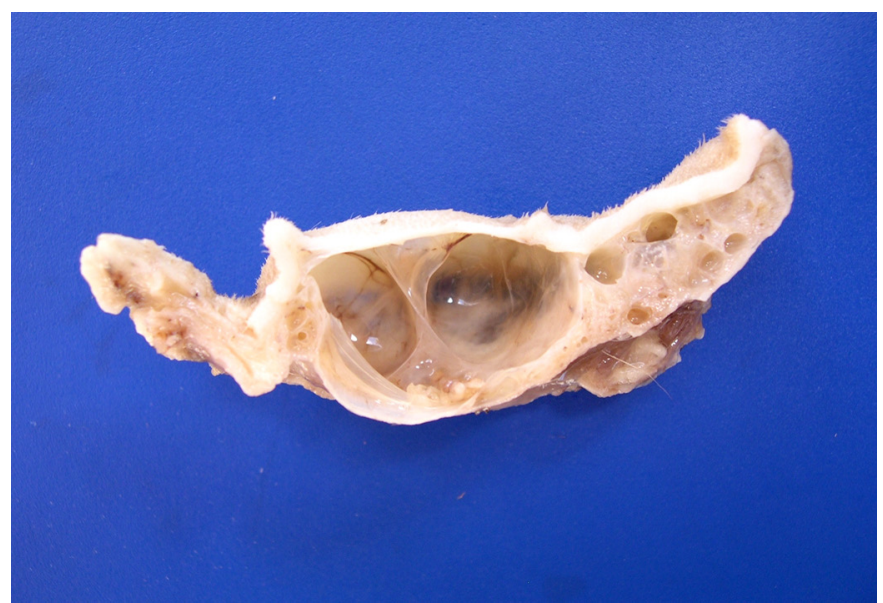

Fig.6. Fragmento de cadeia mamária com displasia cística contendo múltiplos cistos de variados tamanhos.

\section{DISCUSSÃO}

A prevalência de tumores de mama na população de gatos estudados aproxima-se dos 12\%, como encontrado por Dorn et al. (1968). Cotchin (1952), em um estudo sobre tumores em gatos, concluiu que o sistema mais acometido por tumores era o tegumentar, seguido pelo alimentar e reprodutor feminino (incluindo glândula mamária). Anos mais tarde outros autores demonstraram que em gatas o tumor mamário é o terceiro mais frequente, após os tumores cutâneos e hemolinfopoéticos (Dorn et al. 1968, Hayes et al. 1981). Porém, neste estudo há significativas diferenças, os resultados revelam que os tumores cutâneos são os mais prevalentes, seguidos pelos tumores de mama e pelos tumores do sistema hemolinfopoéticos.

A elevada porcentagem dos neoplasmas mamários em relação aos outros tumores diagnosticados no LPV-UFSM poderia ser atribuída a dois motivos. 0 primeiro é que o método de avaliação dos neoplasmas hemolinfopoéticos é feito primariamente através do uso da punção aspirativa por agulha fina (PAAF). Como a PAAF não é utilizada como ferramenta diagnóstica de rotina no LPV-UFSM, o número de diagnósticos de linfomas/leucemias torna-se menor, com consequen- 
te elevação dos índices percentuais dos outros neoplasmas. O segundo motivo da observação de baixo número de tumores hemolinfopoéticos, baseia-se na hipótese da pouca circulação do vírus da imunodeficiência felina (FIV) e do vírus da leucemia felina ( $\mathrm{FeLV}$ ) na região de estudo. Visto que, alguns tumores hemolinfopoéticos podem estar até 70\% associados à infecção por FIV e FeLV (Fighera \& Graça 2011). Entretanto, ainda não existem estudos regionais que sustentam essa hipótese. A causa do elevado número de tumores de pele deve-se principalmente à elevada taxa de diagnósticos de carcinoma de células escamosas (CCE), sendo a alta incidência solar um dos principais agravantes para o desenvolvimento desse neoplasma (Goldschmidt \& Hendrick 2002).

Os neoplasmas mamários das gatas têm grande semelhança com a etiologia hormonal dos tumores de mama em mulheres e em cadelas (Hayden et al. 1989, Hellmén et al. 1993, Russo \& Russo 1995). Entretanto, no presente estudo não foi possível estabelecer qualquer ligação entre o efeito protetor da ovário-histerectomia $(\mathrm{OH})$ nas gatas, devido à falta dessa informação na maioria dos protocolos. Sabe-se que, assim como ocorre em outras espécies, a taxa hormonal tem uma grande influência no desenvolvimento de tumores mamários em gatas e que, como em cadelas, a $\mathrm{OH}$ tem um papel importante na redução do risco de carcinoma mamário felino (Dorn et al. 1968, Hayes \& Mooney 1985, Overley et al. 2005). A proteção conferida pela $\mathrm{OH}$ é de $91 \%$ quando o procedimento for realizado antes dos seis meses de idade, $86 \%$ quando realizado de sete a 12 meses e de apenas $11 \%$ quando o animal tem 13 a 24 meses. Não são observados benefícios na prevenção de carcinoma mamário felino quando a OH é executada após 24 meses de idade (Overley et al. 2005).

A administração de fármacos contraceptivos obteve números pouco significativos neste estudo devido à ausência de dados ou possível ocultação por parte dos proprietários sobre a administração dessas substâncias. Em gatas, somente a administração regular de progesterona foi associada a um significativo aumento no risco de desenvolvimento de tumores mamários benignos e malignos (Misdorp et al. 1991). Com relação às alterações não neoplásicas, o segundo diagnóstico mais encontrado neste estudo, quase a metade dos proprietários afirmaram a administração de contraceptivos. Isso vem ao encontro do fato de que essas lesões na mama de gatas, particularmente a hiperplasia fibroepitelial, estão associadas com progesterona endógena ou exógena (Allen 1973, Misdorp et al. 1999).

A relação benigno:maligno de 1:14,27 encontrada nesse estudo, destaca-se quando comparado ao resultado de $1: 2,75$ encontrado em um estudo realizado no LPV-UFSM, em cadelas (Oliveira Filho et al. 2010). Quanto ao diagnóstico morfológico, os neoplasmas malignos foram os mais prevalentes, sendo o carcinoma o mais frequentemente diagnosticado. Os tumores não neoplásicos foram mais prevalentes do que os neoplasmas benignos concordando com vários estudos realizados nos últimos anos em gatas (Travassos 2004, Spader 2009, Costa 2010) e discordando de estudos realizados em cadelas, onde os neoplasmas benignos são mais prevalentes do que tumores não neoplásicos (Hellmén et al. 1993, Oliveira Filho et al. 2010). Os resultados obtidos neste estudo sugerem que os tumores mamários não neoplásicos em gatas são mais comuns que em cadelas e que, quando neoplásicos, esses são mais frequentemente malignos em gatas do que em cadelas.

As gatas SRD, assim como no estudo realizado por Silva (2010), foram as mais acometidas por tumores mamários. Já com relação às raças puras, os resultados concordam com muitos autores que afirmam uma maior frequência da raça Siamesa (Hayes et al. 1981, Hayes \& Mooney 1985, Silva 2010). Porém, esses resultados diferem de Costa (2010) e Travassos (2004) por que em ambos os estudos, a raça Europeu Comum foi a mais prevalente, seguida da raça Siamesa (Travassos 2004) e da Persa (Costa 2010). Essas variações devem-se, possivelmente às diferenças na população felina de cada região.

A faixa etária no momento do diagnóstico foi semelhante à encontrada em outros estudos, sendo as gatas idosas mais acometidas (Hayes et al. 1981, Castagnaro et al. 1998, Travassos 2004, Costa 2010). Das gatas jovens apenas uma tinha neoplasma, sendo o restante dos diagnósticos morfológicos tumores não neoplásicos. Dados esses muito semelhantes aos encontrados por Allen (1973).

A glândula mamária mais afetada nessa espécie ainda não foi bem definida, sendo encontradas muitas divergências entre diversos estudos. Para Brondey (1970) todas as glândulas mamárias de gatas possuem igual susceptibilidade para desenvolvimento de tumores mamários. Neste estudo, o grupo mamário G1 foi mais acometido do que o G2, concordando com Costa (2010) e divergindo de outros resultados encontrados em gatas (Travassos 2004, Amorim et al. 2006) e em cadelas (Hayes et al. 1981, Oliveira Filho et al. 2010), nas quais as glândulas distais (G2) foram as mais atingidas. Numerosos casos neste estudo tinham ambos os grupos afetados, demonstrando assim que é relativamente comum na espécie felina a existência de múltiplos tumores mamários (Hayes \& Mooney 1985, Misdorp 2002). Quando apenas uma glândula é afetada, deve levar-se em consideração a vasculatura linfática normal dos gatos, que possui características típicas dessa espécie, como na drenagem das glândulas abdominal cranial e abdominal caudal, que podem ter comunicação tanto com linfonodos craniais como com linfonodos caudais (Raharison \& Sautet 2007). Assim, alguns estudos sugerem que gatas que são submetidas a cirurgias radicais tem um maior intervalo livre da doença, mais do que as gatas tratadas com métodos conservativos, contudo nenhum dos métodos altera o tempo de sobrevivência (McEwen et al. 1984).

0 tamanho dos tumores é um dos mais importantes fatores clínicos avaliados para o prognóstico dos neoplasmas mamários de mulheres, cadelas e gatas (Misdorp \& Hart 1976, Hellmén et al. 1993). Embora haja uma maior quantidade de T1, o que deveria indicar um melhor prognóstico (Else \& Hannant 1979, Misdorp 2002), neste estudo observou-se que, desses, $82,97 \%$ eram carcinomas, principalmente com padrão túbulo-papilar. Diferente dos resultados encontrados por Oliveira Filho et al. (2010) em cadelas, onde apenas $66 \%$ dos tumores classificados como $\mathrm{T} 1$ eram neoplasmas malignos. Isso demonstra a necessidade da implementação de outros métodos prognósticos para predizer o comportamento biológico dos tumores mamários em gatas, além do fator tamanho. 
Muitos autores descrevem o envolvimento de linfonodos como sendo um importante fator de prognóstico (Hellmén et al. 1993, Misdorp 2002, Queiroga \& Lopez 2002). A avaliação dos linfonodos no presente estudo não obteve resultados significativos. Este fato deve-se, possivelmente, à maioria dos protocolos terem origem de biópsias, onde a excisão dos linfonodos ocorre, normalmente, apenas quando o seu envolvimento é clinicamente evidente.

Com relação às metástases, os resultados são semelhantes aos encontrados em alguns estudos, onde o pulmão é o órgão mais acometido. Podendo ser encontradas também na pleura, fígado, baço, rins, pele, esqueleto, encéfalo, glândulas adrenais e coração (Hayes et al. 1981).

A ulceração também tem sido usada como um fator prognóstico, sendo descrita em cães, principalmente nos neoplasmas malignos (Queiroga \& Lopes 2002, Oliveira Filho et al. 2010). Neste estudo essa lesão esteve associada geralmente a neoplasmas malignos, sendo o carcinoma túbulo-papilar o mais comumente ulcerado. Porém, é importante ressaltar a presença de ulcerações também em alterações não neoplásicas como a hiperplasia fibroepitelial. Não foi possível estabelecer se as ulcerações foram devido à abrasão no solo por seu grande tamanho, ou por seu acelerado crescimento invasivo e destrutivo.

\section{CONCLUSÕES}

Através do presente estudo concluiu-se que, na área de abrangência do LPV-UFSM, os gatos são afetados, em ordem decrescente de prevalência, por tumores de pele, mamários e hemolinfopoéticos, revelando assim a importância dos tumores de mama nessa espécie.

A alta prevalência dos neoplasmas mamários malignos em relação aos benignos, a variação no tamanho dos neoplasmas malignos e a presença de ulcerações também em alterações não neoplásicas, são resultados que reafirmam a importância da avaliação de um conjunto de fatores prognósticos, tanto no histórico, como no exame clínico, associado ao diagnóstico histopatológico, remetendo assim a um programa terapêutico adequado.

\section{REFERÊNCIAS}

Allen H.L. 1973. Feline mammary hypertrophy. Vet. Pathol. 10:501-508.

Amorim F.V., Souza H.J.M., Ferreira A.M.R. \& Fonseca A.B.M. 2006. Clinical, cytological and histopathological evaluation of mammary masses in cats from Rio de Janeiro, Brazil. J. Feline Med. Surg. 8:379-388.

Brondey R.S. 1970. Canine and feline neoplasia. Adv. Vet. Sci. Comp. Med. 14:309-354.

Castagnaro M., Casalone C., Bozzetta E., De Maria R., Biolatti B. \& Caramelli M. 1998. Tumour grading and the one year post-surgical prognosis in feline mammary carcinoma. J. Comp. Pathol. 119:263-275.

Costa M.M. 2010. Estudo epidemiológico e anatomo-patológico de tumores mamários na cadela e na gata. Dissertação de Mestrado Integrado em Medicina Veterinária, Faculdade de Medicina Veterinária, Universidade Técnica de Lisboa, Lisboa. 135p.

Cotchin E. 1952. Neoplasms in cats. Proc. Roy. Soc. Med. 45:17-20.

Dorn E.R., Taylor D., Frye F.L. \& Hibbard H.H. 1968. Survey of animal neoplasms in Alameda and Contra Costa County, California. I. Methodology and description of cases. J. Natl. Cancer Inst. 40:295-305.

Else R.W. \& Hannant D. 1979. Some epidemiological aspects of mammary neoplasia in the bitch. Vet. Rec. 194:296-304.
Fighera R.A. \& Graça D.L. 2011. Sistema hematopoiético, p.337-422. In: Santos R.L. \& Alessi A.C. (Eds), Patologia Veterinária. Roca, São Paulo.

Hayden D.W., Barnes D.M. \& Johnson K.H. 1989. Morphologic changes in the mammary gland of megestrol acetate treated and untreated cats: a retrospective study. Vet. Pathol. 26:104-113.

Hayes A.A. \& Mooney S. 1985. Feline mammary tumors. Vet. Clin. North Am., Small Anim. Pract. 15:513-520.

Hayes H.M., Milne K.L. \& Mandell C.P. 1981. Epidemiological studies of feline mammary carcinoma. Vet. Rec. 108:476-479.

Hellmén E., Bergstrom R., Holmberg L., Spångberg I.B., Hansson K. \& Lindgren A. 1993. Prognostic factors in canine mammary tumors: A multivariate study of 202 consecutive cases. Vet. Pathol. 30:20-27.

Hampe J.F. \& Misdorp W. 1974. Tumours and dysplasias of the mammary gland, p.111-133. In: International Histological Classification of Tumors of Domestic Animals. Bull. 50, World Health Organization, Geneva.

Goldschmidt M.H. \& Hendrick M.J. 2002. Tumors of the skin and soft tissues, p.44-117. In: Meuten D.J. (Ed.), Tumors in Domestic Animals. $4^{\text {th }}$ ed. Iowa State Press, Ames.

McEwen E.G., Hayes A.A., Harvey H.J., Patnaik A.K., Mooney S. \& Passe S. 1984. Prognostic factors for feline mammary tumors. J. Am. Vet. Med. Assoc. 185:201-204.

Misdorp W. \& Hart A.A.M. 1976. Prognostic factors in canine mammary cancer. J. Natl Cancer Inst. 56:779-786.

Misdorp W. \& Weijer K. 1980. Feline mammary carcinoma. Am. J. Pathol. 98:573-576.

Misdorp W., Romijn A. \& Hart A.A.M. 1991. Feline mammary tumors: A case control study of hormonal factors. Anticancer Res. 11:1793-1798.

Misdorp W., Else R.W., Hellmén E. \& Lipscomb T.P. 1999. Histological classification of mammary tumors of the dog and cat, p.63-64. In: Ibid. (Eds), International Histological Classification of Tumors of Domestic Animals. Second Series, Vol.7. World Health Organization, Geneva, Switzerland.

Misdorp W. 2002. Tumors of the mammary gland, p.575-606. In: Meuten D.J. (Ed.). Tumors in Domestic Animals. $4^{\text {th }}$ ed. Iowa State Press, Ames.

Oliveira Filho J.C., Kommers G.D., Masuda E.K., Marques B.M.F.P.P., Fighera R.A., Irigoyen L.F. \& Barros C.S.L. 2010. Estudo retrospectivo de 1.647 tumores mamários em cães. Pesq. Vet. Bras. 30:177-185.

Overley B., Shofer F.S., Goldschimitd M.H., Sherer D. \& Sorenmo K.U. 2005. Association between ovarihyst erectomy and feline mammary carcinoma. J. Vet. Intern. Med. 19:560-563.

Pérez-Alenza M.D., Jiménez A., Nieto A.I. \& Pena L. 2004. First description of feline inflammatory mammary carcinoma: clinicopathological and immunohistochemical characteristics of three cases. Breast Cancer Res. 6:300-307.

Queiroga F. \& Lopes C. 2002. Tumores mamários caninos, pesquisa de novos factores de prognóstico. RPCV 97:119-127.

Raharison F. \& Sautet J. 2007. The topography of the lymph vessels of mammary glands in female cats. Anat. Histol. Embryol. 36:442-452.

Russo J. \& Russo I.H. 1995. The etiopathogenesis of breast cancer prevention. Cancer Lett. 90:81-89.

Silva M.N.S. 2010. Avaliação de neoplasias mamárias malignas felinas quanto aos aspectos histopatológicos e a expressão imuno-histoquímica de Ki-67 e caspase 3 clivada. Dissertação de Mestrado em Anatomia Patológica Humana e Veterinária, Universidade Federal Fluminense, Niterói. 93p.

Spader M.B. 2009. Estudo epidemiológico, classificação histológica e fatores prognósticos pela técnica de quantificação das AgNORS em tumores mamários felinos. Dissertação de Mestrado em Patologia Animal, Programa de Pós-Graduação em Veterinária, Universidade Federal de Pelotas, Pelotas. $57 \mathrm{p}$.

Travassos F.A.G.S. 2004. Lesões mamárias felinas: contributo para sua caracterização biopatológica. Tese de Doutorado, Departamento de Ciências Veterinárias, Universidade de Trás-os-Montes e Alto Douro, Vila Real. 251p.

Weijer K. \& Hart A.A.M. 1983. Prognostic factors in feline mammary carcinoma. J. Natl. Cancer Inst. 70:709-716. 